\title{
ORIGINAL ARTICLE Radiotherapy-induced miR-223 prevents relapse of breast cancer by targeting the EGF pathway
}

\author{
L Fabris $^{1,9}$, S Berton ${ }^{1,2,9}$, F Citron ${ }^{1}$, S D'Andrea ${ }^{1}$, I Segatto ${ }^{1}$, MS Nicoloso ${ }^{1,3}$, S Massarut ${ }^{4}$, J Armenia ${ }^{1}$, G Zafarana ${ }^{2}$, S Rossi ${ }^{3,10}$, C Ivan ${ }^{3}$,

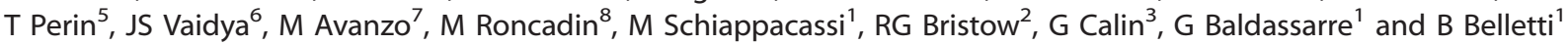

In breast cancer (BC) patients, local recurrences often arise in proximity of the surgical scar, suggesting that response to surgery may have a causative role. Radiotherapy (RT) after lumpectomy significantly reduces the risk of recurrence. We investigated the direct effects of surgery and of RT delivered intraoperatively (IORT), by collecting irradiated and non-irradiated breast tissues from BC patients, after tumor removal. These breast tissue specimens have been profiled for their microRNA (miR) expression, in search of differentially expressed miR among patients treated or not with IORT. Our results demonstrate that IORT elicits effects that go beyond the direct killing of residual tumor cells. IORT altered the wound response, inducing the expression of miR-223 in the peritumoral breast tissue. miR-223 downregulated the local expression of epidermal growth factor (EGF), leading to decreased activation of EGF receptor (EGFR) on target cells and, eventually, dampening a positive EGF-EGFR autocrine/paracrine stimulation loop induced by the post-surgical wound-healing response. Accordingly, both RT-induced miR-223 and peri-operative inhibition of EGFR efficiently prevented BC cell growth and reduced recurrence formation in mouse models of BC. Our study uncovers unknown effects of RT delivered on a wounded tissue and prompts to the use of anti-EGFR treatments, in a peri-operative treatment schedule, aimed to timely treat BC patients and restrain recurrence formation.

Oncogene (2016) 35, 4914-4926; doi:10.1038/onc.2016.23; published online 15 February 2016

\section{INTRODUCTION}

Wound healing and tumorigenesis are processes that rely on similar molecular mechanisms, ${ }^{1}$ including the local production of growth factors and cytokines that can eventually influence the behavior of residual and/or attract dormant or circulating tumor cells. $^{2}$ In breast cancer (BC) clinical and experimental evidence support the notion that the inflammatory and wound-healing responses triggered by the act of surgery may modify the growth kinetics of micrometastasis, eventually supporting the survival and the local regrowth of residual BC cells. ${ }^{3-9}$

Breast-conserving surgery followed by external beam radiation therapy represents the standard of care for the majority of BC patients. ${ }^{10}$ Recent clinical trials have demonstrated that a single application of radiotherapy delivered intraoperatively (IORT) to the tumor bed after removal of the tumor mass may be non-inferior to 6 weeks of external beam radiation therapy in preventing the formation of local recurrences in selected BC patients. ${ }^{11,12}$

IORT offered the unique opportunity to study the early effects of radiotherapy (RT) on human tissues in vivo. Our previous study has demonstrated that IORT is able to modify the post-surgical microenvironment, by altering the composition of fluids that normally accumulate in the tumor bed after surgery (drainage wound fluids, WF). ${ }^{4}$ Yet, how RT impinges on the molecular composition of these WF is still unknown.
MicroRNAs (miR) are small, non-coding RNA molecules that regulate gene expression post-transcriptionally. They are expressed in a tissue-specific manner ${ }^{13}$ and can be secreted and stably expressed in animal serum/plasma. ${ }^{14}$ They have emerged as essential players in the regulation of morphogen and growth factor bioavailability, through non-cell-autonomous mechanisms, ${ }^{15}$ as well as in governing the crosstalk between tumor cells and their microenvironment. ${ }^{16}$ Several studies have shown that ionizing radiations induce changes in miR expression profiles in cells and in preclinical models. ${ }^{17}$ Yet, the immediate effects of RT on miR expression and their significance in vivo, if any, are unknown.

Here, we investigate the modulation of miR by RT in human tissues in vivo. By examining miR expression profiles in breast tissues from IORT-treated BC patients after lumpectomy, we highlight the importance of the post-surgical tumor-stroma crosstalk in dictating clinical outcome.

\section{RESULTS}

Intraoperative RT induces miR-223 expression in peri-tumoral breast tissue

We have previously demonstrated that irradiation (IR) of wounded peri-tumoral breast tissue with IORT changed the molecular

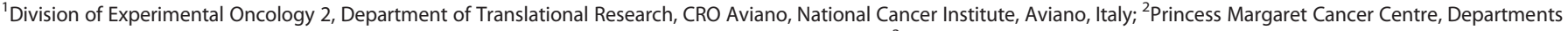

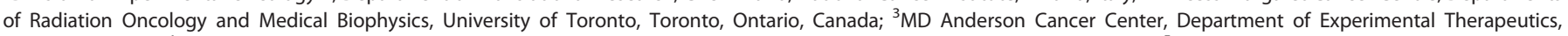

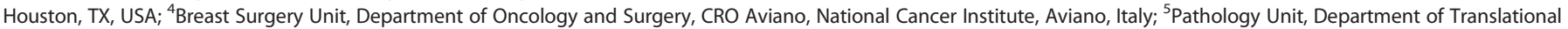

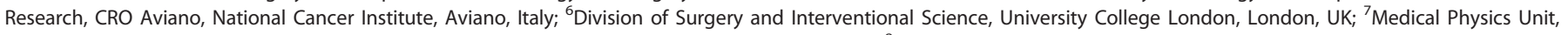

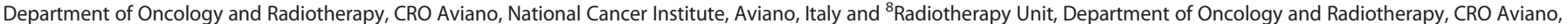

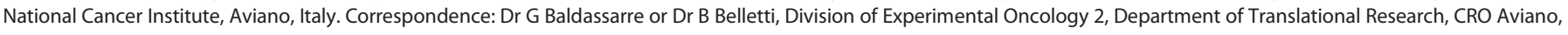
National Cancer Institute, Via Gallini 2, Aviano 33081, Italy.

E-mail: gbaldassarre@cro.it or bbelletti@cro.it

${ }^{9}$ These authors contributed equally to this work.

${ }^{10}$ Current address: Swiss Institute of Bioinformatics, Quartier Sorge, Bâtiment Génopode, CH-1015 Lausanne, Switzerland.

Received 9 June 2015; revised 4 November 2015; accepted 18 December 2015; published online 15 February 2016 
composition and biological activity of WF drained from BC patients for $24 \mathrm{~h}$ post surgery. ${ }^{4}$ To get more insights into the biological modifications induced by IORT, we collected specimens from BC patients enrolled in the TARGIT-A trial, undergoing lumpectomy followed or not by IORT treatment. ${ }^{11,12}$ We collected two specimens of normal peri-tumoral mammary tissue from 29 patients (Supplementary Table S1). The first specimen was collected immediately after removal of the primary tumor (specimen at surgery) and the second one $30 \mathrm{~min}$ after surgery (specimen post surgery) or after IORT treatment (specimen post IORT; Figure 1a). Pathological scrutiny of breast tissue sampling was performed to ensure that the mammary gland was adequately and similarly represented in all specimens (Supplementary Figure S1). IORT did not induce macroscopic alteration of the mammary gland architecture and composition, with respect to the samples collected after surgery (Supplementary Figure S1). From the same patients, we also collected WF to be used as a surrogate model of wound response in in vitro experiments.

Given the possible dual action of miR in both cell-autonomous and non-cell-autonomous manner, we decided to perform a miR microarray in seven specimens/group of patients, used as discovery set. Comparative analysis of the two specimens collected from each patient, at and after surgery, demonstrated that the act of surgery modified the expression of 74 miRs (Supplementary Figure S2A and Supplementary Table S2). The same analysis performed before and after IORT delivery indicated significant changes in the expression of nine miRs (Figure $1 \mathrm{~b}$ and Supplementary Table S3). Forty-one miRs were differentially expressed between the IORT group versus

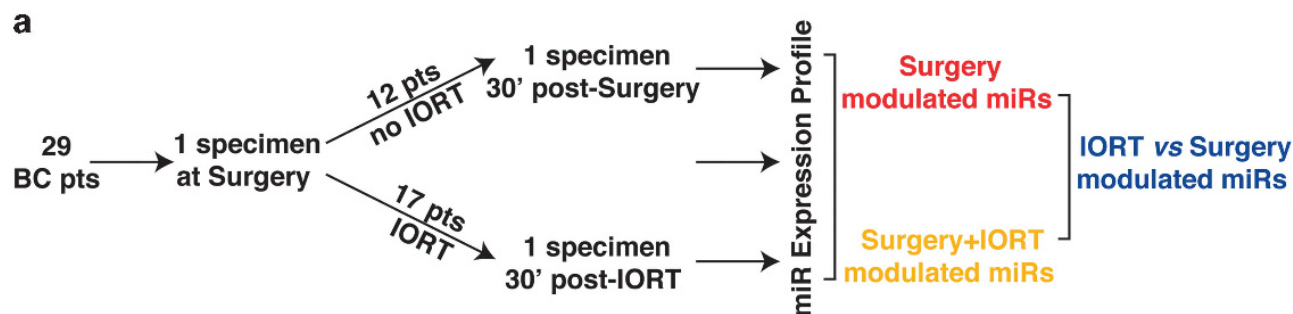

b

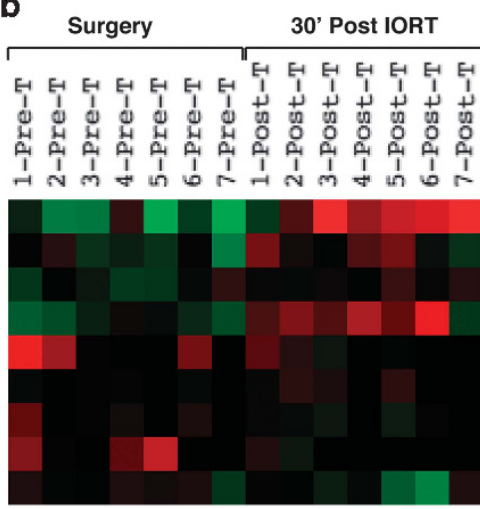

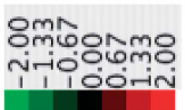

hsa-miR -223

hsa-miR -126 *

hsa-miR-1255a-P

hsa-mir-565-P

hsa-miR-202

hsa-miR $-155^{*}$

hsa-miR -424 *

hsa-mir-675b-P

hsa-miR-923-P
C

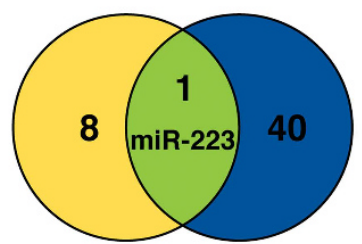

Surgery + IORT modulated miRs

IORT vs Surgery modulated miRs

IORT modulated miRs
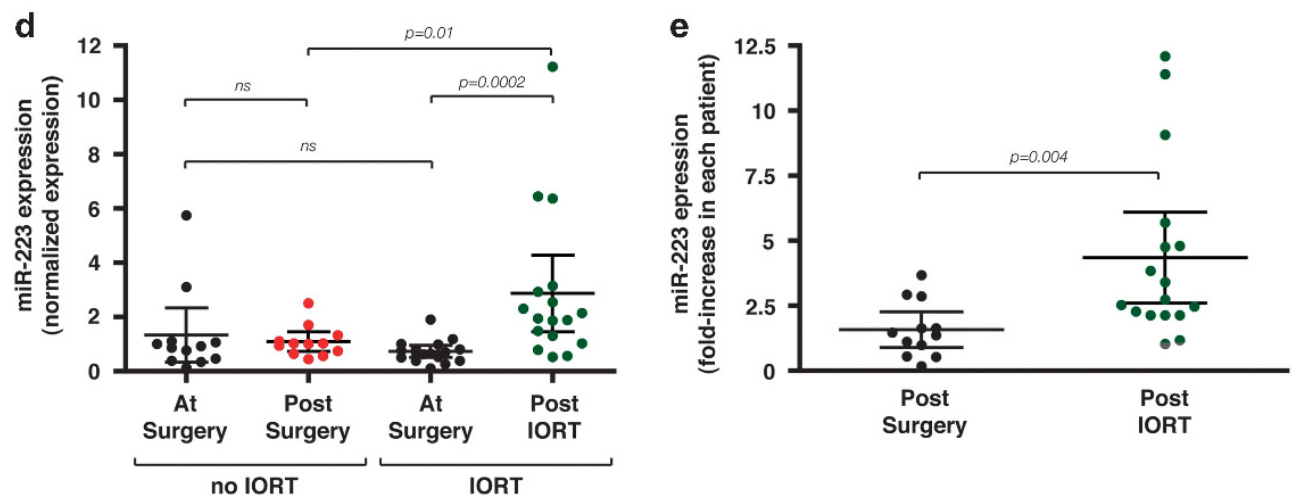

Figure 1. IORT treatment modifies miR expression in peri-tumoral microenvironment. (a) Schematic representation of the experimental design. Two specimens of peri-tumoral breast tissue were collected from 29 BC patients undergoing surgery. Among these patients, 12 underwent only surgery and 17 also underwent IORT. From each patient, one specimen was collected right after surgery ('at surgery') and one 30 min post surgery or 30 min post IORT. (b) Heat map of differentially expressed miRs in paired samples of the IORT-treated patients. (c) Venn diagram of miRs differentially expressed in paired samples of the IORT-treated patients (yellow) and in samples collected post surgery with respect to post-IORT (blue). miR-223 is differentially expressed in both comparisons (green). (d, e) qRT-PCR of miR-223 expression in the groups described in a, expressed as normalized for U6 levels (d) or as fold increase in paired samples from each patient. (e) Graphs report the median value ( \pm s.d.) of samples in each group. Statistical significance is reported in each graph and was calculated using the MannWhitney test. 
the group who received only surgery (Figure 1c, Supplementary Figure S2B and Supplementary Table S4). Among these, miR-223 was the most highly and significantly modified (Figure 1b, Supplementary Figure S2 and Supplementary Tables S2 and S4) and was the only miR specifically modulated by IORT (Figure 1c). We validated this result in a wider collection of specimens $(n=29)$ by quantitative reverse transcription-PCR, highlighting that not only miR-223 expression level was significantly augmented in the IORT group (Figure 1d), but also that it was specifically increased in each single patient, comparing the specimen collected at surgery with that collected 30 min after IORT (Figure 1e).

Thus, miR-223 expression is induced by IR in the mammary tissue of patients, following surgical removal of $B C$ mass. a

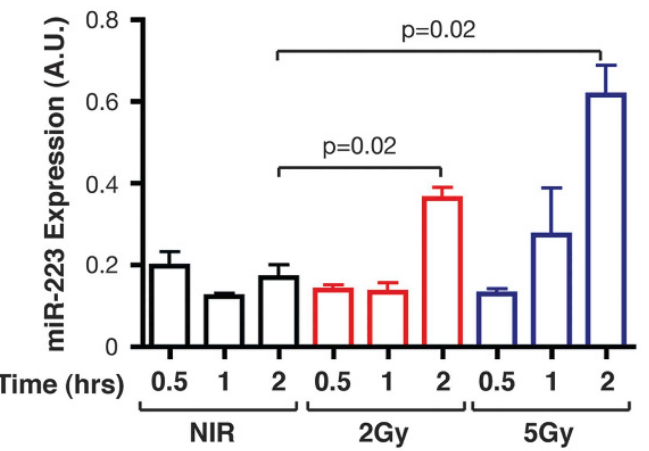

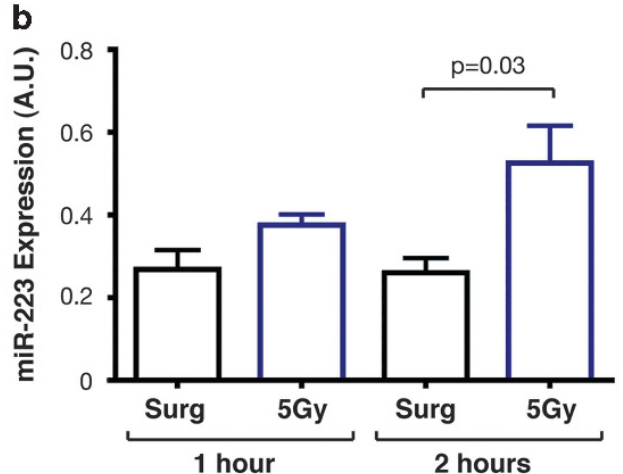

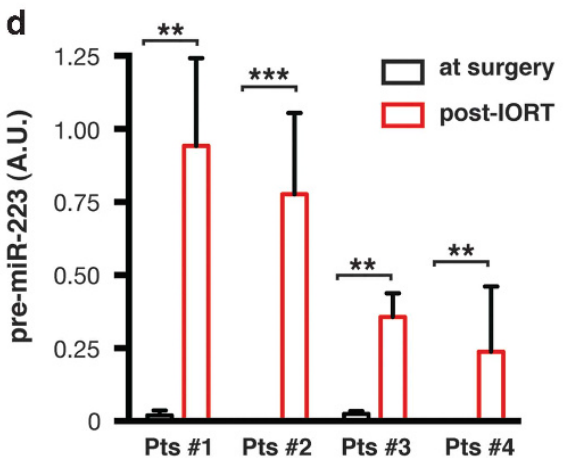

C

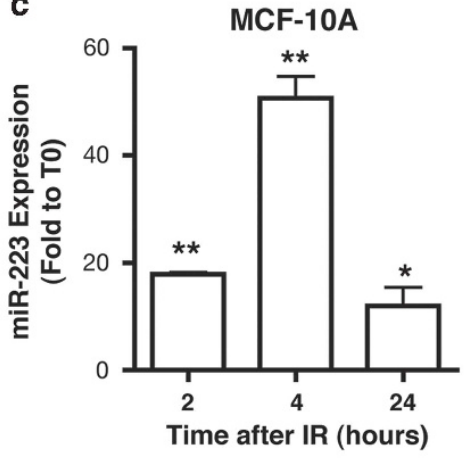

MDA-MB-231

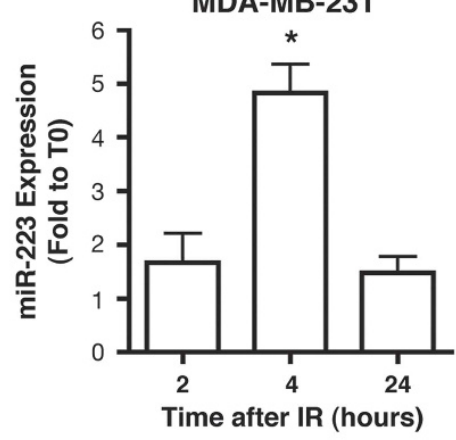

f

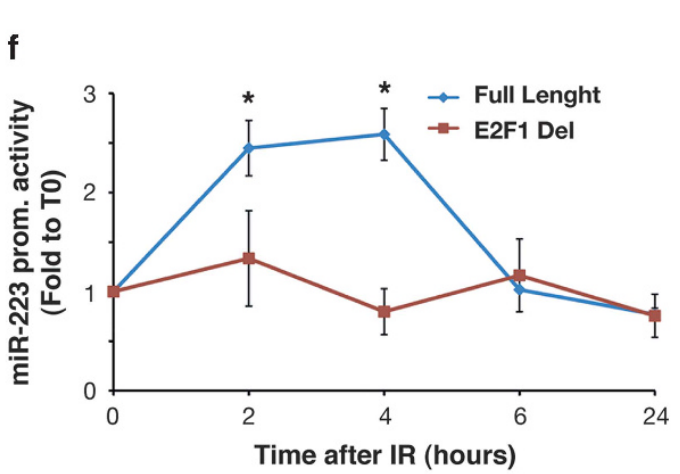

9
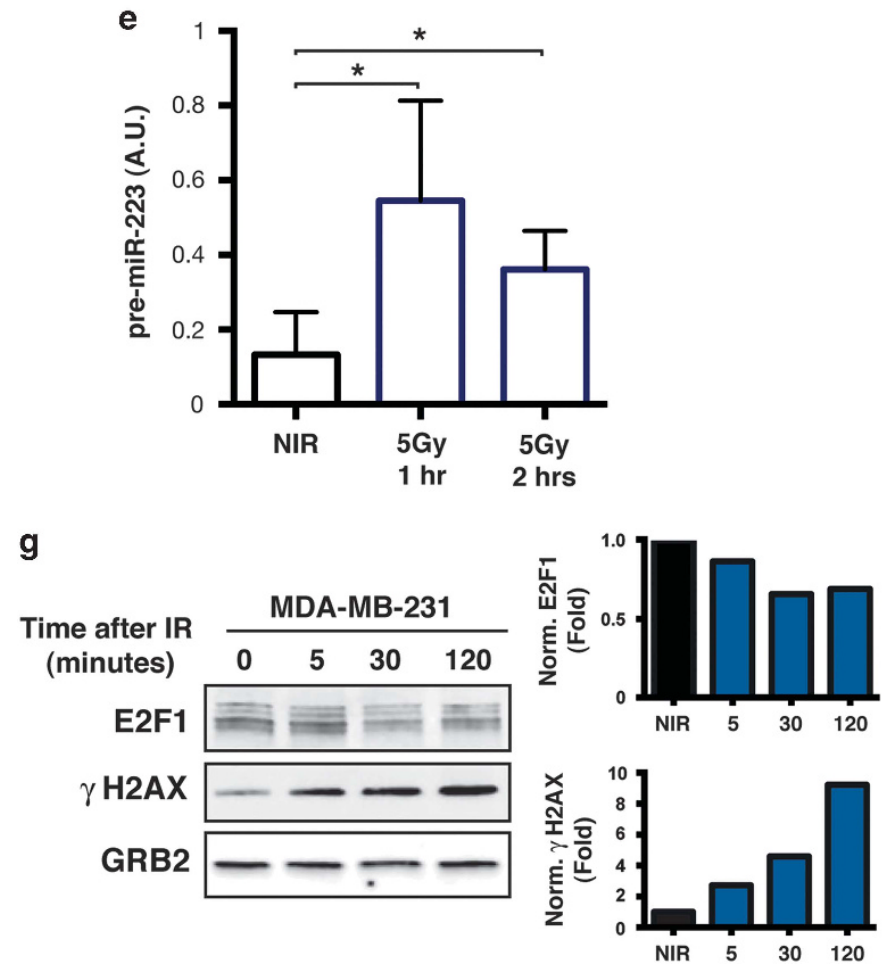

h

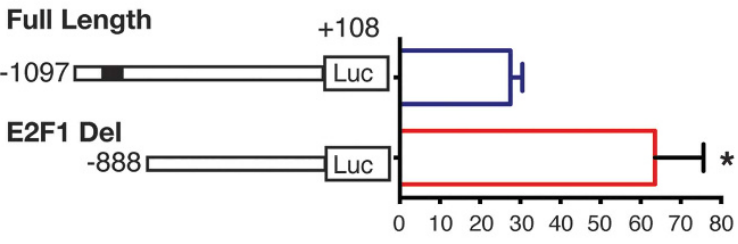

miR-223 promoter activity 
RT induces miR-223 expression also in mouse breast tissue in vivo, and in breast epithelial cells in vitro

In order to test whether modulation of miR-223 was a common event following breast tissue IR, we set up a mouse model of IORT treatment, using the Intrabeam device (Carl Zeiss, Oberkochen, Germany; Supplementary Figure S3). Mice were all subjected to a surgical procedure and then irradiated in the mammary gland (or not, in the control group). Mice were then killed $0.5,1$ and $2 \mathrm{~h}$ after IORT ( 2 or $5 \mathrm{~Gy}$ at $5 \mathrm{~mm}$ from the applicator) or after surgery alone. Levels of miR-223 increased in a dose- and time-dependent manner in IORT-treated mouse breast tissue, peaking at $2 \mathrm{~h}$ after RT (Figures 2a and b). As observed in the tissues from BC patients, the act of surgery alone did not modify miR-223 expression (Figure 2b). Similar results were obtained by irradiating in vitro mammary epithelial cells, such as the MCF-10A and the MDAMB-231 cell lines (Figure 2C). MCF-10A, a model of non-malignant mammary epithelial cells, expressed much higher levels of miR-223 than MDA-MB-231 basal-like BC cells, both at basal level and after IR, possibly reflecting the tissue source that we observed in samples from patients (normal peri-tumoral mammary gland).

Thus, miR-223 expression is induced by IR also in mouse mammary gland in vivo, and in mammary non-malignant and cancer cells in vitro.

The kinetics of miR-223 expression, observed both in vivo and in vitro, suggested that IR could induce miR-223 at transcriptional level. Accordingly, pre-miR-223 expression was upregulated by IORT, both in specimens from BC patients (Figure 2d) and in mouse mammary glands (Figure 2e). Moreover, we measured the activity of miR-223 full-length promoter in irradiated MDA-MB-231 cells and observed that IR induced a time-dependent increase in luciferase activity (Figure $2 \mathrm{f}$, blue line). It has been recently reported that the E2F1 transcription factor has a role in controlling miR-223 expression ${ }^{18,19}$ and, interestingly, E2F1 protein levels decreased within $30 \mathrm{~min}$ in IR cells (Figure $2 \mathrm{~g}$ ). We thus tested whether E2F1 could be involved in our context. We measured the activity of miR-223 full-length promoter and of a mutated promoter, deleted for E2F1-binding sites, both in basal conditions and following IR. Although displaying a higher basal activity (Figure 2h), conceivably for lack of E2F1-mediated suppression, the deletion mutant was insensitive to IR (Figure $2 \mathrm{f}$, red line), strongly suggesting that E2F1 represented a critical mediator of IR-induced transcription of miR-223.

miR-223 directly regulates the expression of epidermal growth factor

Data collected so far indicated that IR of breast epithelial cells stimulated miR-223 promoter activity, eventually resulting in the upregulation of miR-223 expression.
To understand the role of miR-223 in the post-surgery context, we searched for potential miR-223 targets, coupling proteomics with bioinformatics analyses. We interrogated six different targetprediction algorithms for potential miR-223 targets among the 187 cytokines tested in WF in our previous study (Figure 3a). ${ }^{4}$ By selecting only miR-223 targets indicated by at least two different algorithms, we identified 24 cytokines. Then, we further selected those cytokines whose expression in WF inversely correlated with miR-223 levels (nine cytokines) and, finally, validated their levels in WF from each patient by ELISA. This approach led to the identification of epidermal growth factor (EGF) and prolactin, as putative targets of miR-223 that were expressed significantly less in WF from IORT-treated than in WF from untreated patients (Figures $3 \mathrm{~b}$ and $\mathrm{c}$ and Supplementary Figure S4). We focused our attention to EGF, which represents a key mediator of BC cell proliferation in response to surgery. ${ }^{9}$ We tested its expression also in a wider panel of WF collected for our previous study. ${ }^{4}$ Overall, by analyzing WF from 56 BC patients, we observed that EGF levels were reduced of about $50 \%$ in WF from IORT-treated patients, with a median expression of $4.71 \mathrm{ng} / \mathrm{ml}$ in untreated patients $(n=27)$ and of $2.5 \mathrm{ng} / \mathrm{ml}$ in IORT-treated patients $(n=29$; $P=0.004$, Figure $3 \mathrm{~b}$ ). Thus, EGF is a potential miR-223 target and its expression levels are reduced in WF from IORT-treated patients.

We next investigated whether EGF represented a bona fide target of miR-223 in mammary epithelial cells. EGF is produced as a long precursor and bioinformatic analyses identified three potential miR-223-binding sites in its transcript; one in the coding region (site 1) and two in the 3'-UTR (site 2 and 3; Figure 3d). Sitedirected mutagenesis of these sequences coupled with luciferase assays demonstrated that miR-223 expression efficiently downregulated luciferase activity of EGF-3'-UTR and that binding sites 1 and 3 were necessary to mediate these effects (Figure $3 e$ ). Consistently, overexpression of miR-223 either in MCF-10A or in different $B C$ cell lines (Figure $3 f$ ) reduced EGF expression and secretion in conditioned media (Figure $3 \mathrm{~g}$ and Supplementary Figure S5A). Importantly, conditioned media harvested from miR-223 overexpressing MDA-MB-453 cells (Supplementary Figure S5B, left panel) stimulated EGF receptor (EGFR) phosphorylation in BT-474 cells less efficiently than conditioned media from control-transfected cells (Supplementary Figure S5B, right panel). Similarly, WF from IORT-treated patients less efficiently induced phosphorylation of EGFR in different cellular models, with respect to WF from control patients (Supplementary Figures S5C and S5D). It has been proposed that phosphorylation of EGFR at Y1068 is necessary for the activation of STAT3 and PI3K/mTOR/p70S6K pathways. $^{20,21}$ Accordingly, lower phosphorylation of Y1068 EGFR was coupled with decreased activation of both STAT3 and p70S6K signaling pathways (Supplementary Figures S5E and F), also in our context. ${ }^{4}$

Figure 2. Irradiation induces miR-223 expression both in vivo and in vitro. (a) qRT-PCR of normalized miR-223 expression in samples from mice treated with IORT, at $2 \mathrm{~Gy}$ or $5 \mathrm{~Gy}$, collected at the indicated time point after the irradiation $(n=3)$. Controlateral non-irradiated (NIR) mammary gland of each mouse was used as a control $(n=6)$. (b) qRT-PCR of normalized miR-223 expression in samples from mice subjected to surgery on the left and surgery plus IORT ( $5 \mathrm{~Gy}$ ) on the right mammary gland, after 1 and $2 \mathrm{~h}$ from irradiation ( $n=4$ for each time point). (c) qRT-PCR of miR-223 (expressed as fold increase over the NIR cells) in MCF-10A mammary epithelial cells (left graph) and in MDA-MB-231 breast cancer cells (right graph) irradiated in vitro with 2 Gy. Data were normalized using U6 expression levels. (d) qRT-PCR of pre-miR-223 expression in paired breast peri-tumoral tissues, described in Figure 1. Specimens were harvested at the moment of surgery, right after tumor removal (at surgery) or after IORT delivery (post IORT). Data were normalized using GAPDH expression levels. (e) qRT-PCR of pre-miR-223 expression in mouse breast tissues described in b. Data were normalized using GAPDH expression levels. (f) Graph reports normalized luciferase activity associated with full-length (blue line) and with E2F1-deleted (E2F1-Del) (red line) miR-223 promoter, transfected in MDAMB-231. Data are expressed as fold increase over the TO of irradiation. Schematic representation of the promoters is shown in $\mathbf{h}$. (g) Western blot analysis of E2F1 expression in MDA-MB-231 cells, irradiated with $2 \mathrm{~Gy}$ and collected at the indicated time points after irradiation. Histone $\gamma \mathrm{H} 2 \mathrm{AX}$ and GRB2 expression were used as a marker of IR efficacy and as loading control, respectively. On the right, graphs report quantification of E2F1 (top panel) and $\gamma \mathrm{H} 2 \mathrm{AX}$ (bottom panel), expressed as fold increase over the NIR sample. (h) Luciferase activity of fulllength and E2F1-Del miR-223 promoters, transfected in MDA-MB-231 irradiated with 2 Gy expressed as fold induction, with respect to normalized NIR cells. The Student's $t$-test or Mann-Whitney test have been used for statistical analysis, as appropriate. Asterisks indicate significant differences, ${ }^{*} P \leqslant 0.05 ;{ }^{* *} P \leqslant 0.01 ;{ }^{* * *} P \leqslant 0.001$. 
a

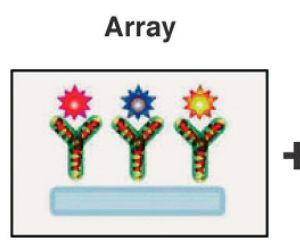

187 Cytokines
6 Prediction Algorithms

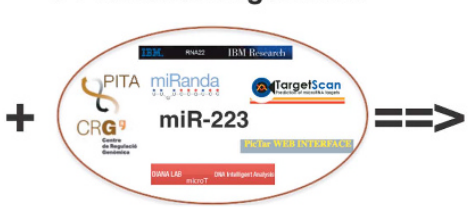

$=24$ Potential

Targets
Check Cytokines Levels

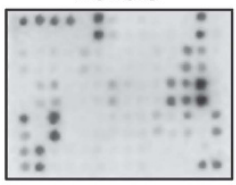

$=9$ Potential

Targets

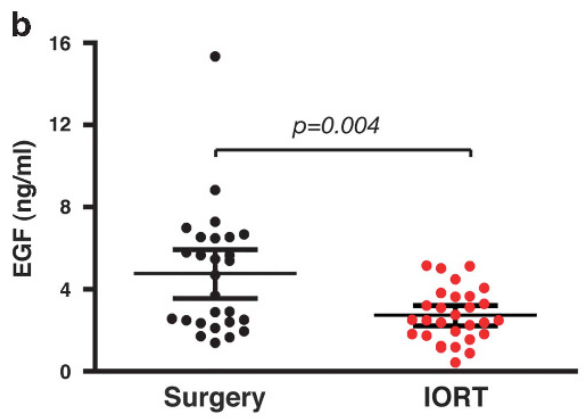

c

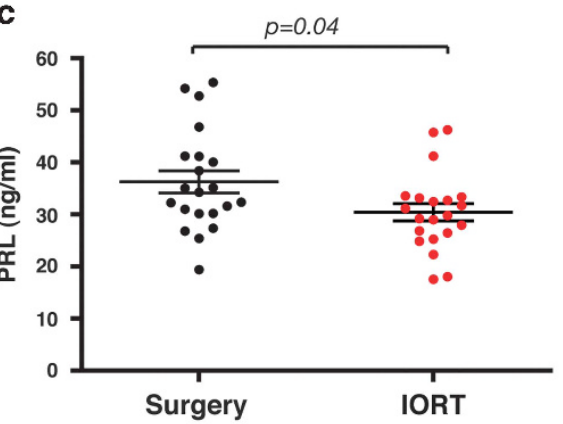

d

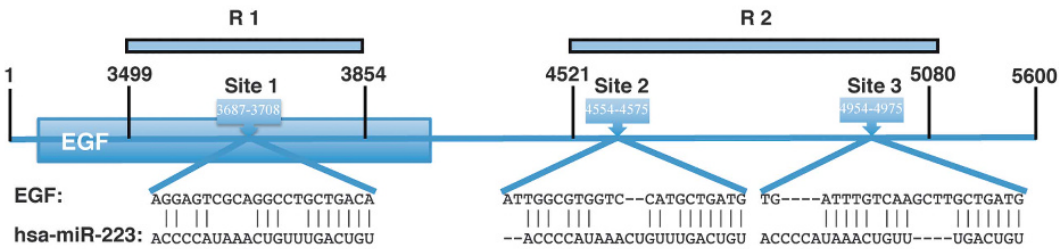

e

Control LuC

R1

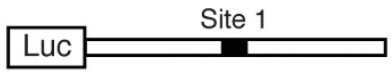

R1-m

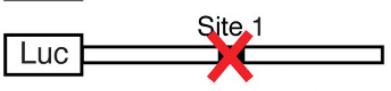

R2

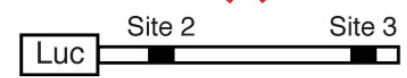

R2-m1

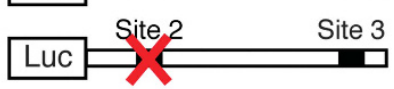

R2-m2
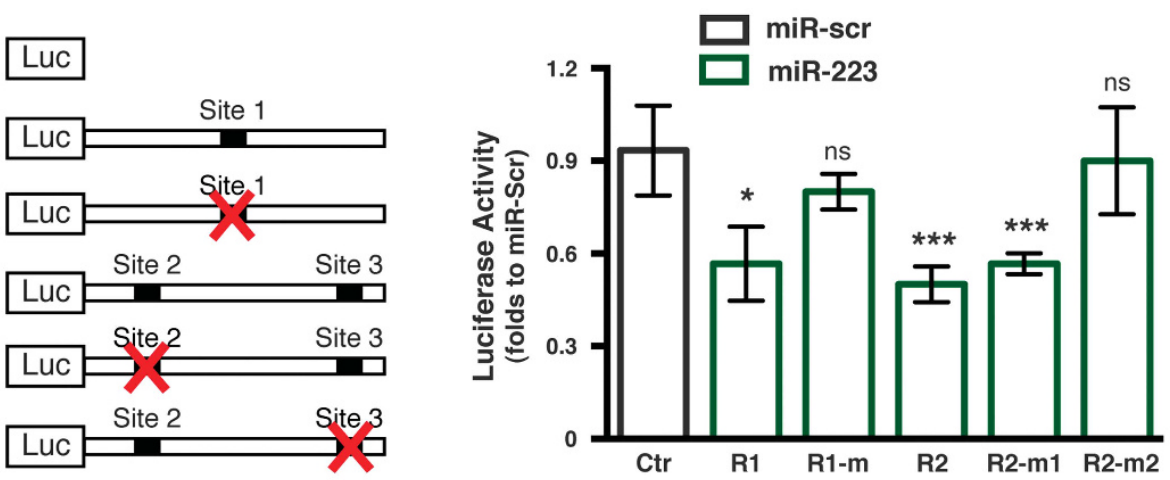

f
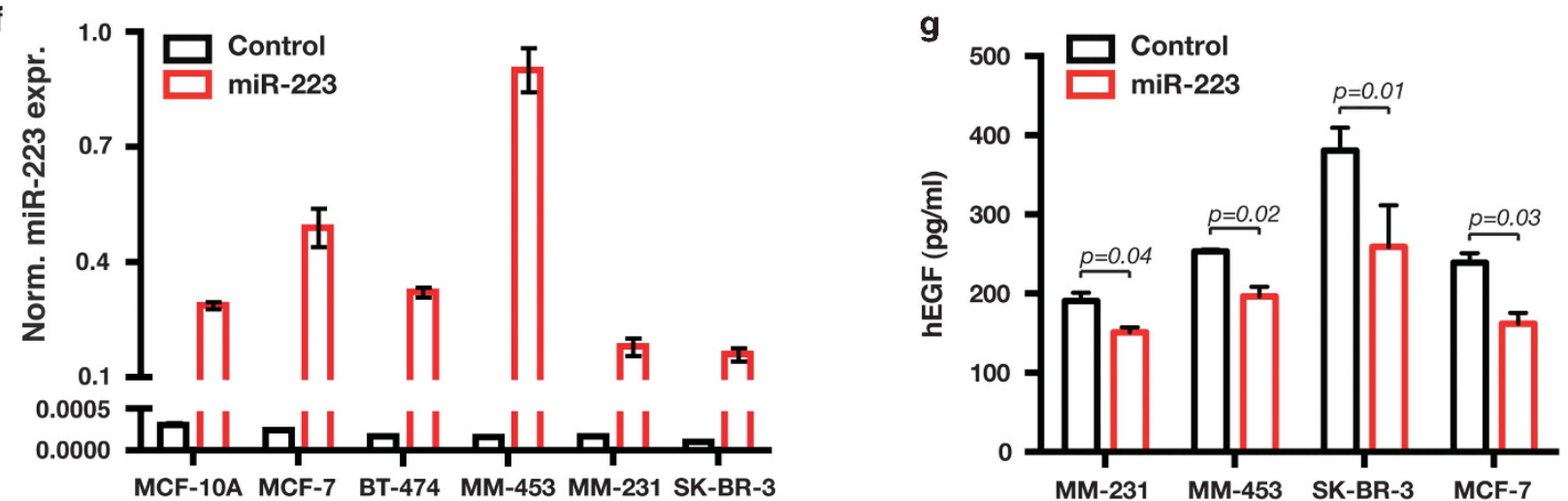

Figure 3. miR-223 controls EGF expression both in vivo and in vitro. (a) Schematic representation of the approach used to identify potential miR-223 targets in WF from control and IORT-treated BC patients. (b, c) ELISA quantification of EGF (b) and prolactin (c) in WF from patients subjected to surgery alone ( $n=27$ for EGF, $n=21$ for prolactin) or surgery plus IORT ( $n=29$ for EGF, $n=21$ for prolactin), as indicated. Statistical significance reported in figure was calculated using the Mann-Whitney unpaired $t$-test. Two-way ANOVA test confirmed the significant differences in EGF expression among groups $(P=0.006)$. (d) Schematic representation of putative miR-223-binding sites in EGF mRNA. (e) On the left side, schematic representation of putative miR-223 seed regions of EGF cloned in their wild-type or mutated form in pGL3 control vector. Graph on the right reports the normalized luciferase activity of each construct in the presence of miR scrambled (black bar) or miR-223 (green bars), expressed as the ratio between miR-223- and miR-scrambled-transfected cells. Control indicates cells transfected with the empty pGL3 vector. Asterisks indicate significant differences respect to the control. (f) qRT-PCR analyses of basal miR-223 expression (black bars) or after transduction with miR-223 lentiviral vector (red bars) in MCF-10A and in the indicated tumor-derived cell lines. miR-223 expression was normalized using U6 levels. (g) ELISA quantification of EGF levels in conditioned media harvested from BC cell lines described in f. The Student's $t$-test or Mann-Whitney test have been used for statistical analysis, as appropriate. Asterisks indicate significant differences, ${ }^{*} P \leqslant 0.05$; $* * P \leqslant 0.01 ; * * * \leqslant 0.001$. 
Overall, we demonstrated that EGF is a bona fide miR-223 target and its reduced expression and secretion in miR-223 overexpressing cells (or in cells stimulated with IORT-WF) is able to reduce EGFR signaling pathway activation.

miR-223 expression hinders WF-induced deregulated growth of BC cells

Stimulation of BC cells with WF from control patients induced a rapid and significant upregulation of EGF expression (Figure 4a), suggesting that this could result in cell proliferation. To test whether miR-223 could impact on this mechanism, we analyzed the proliferative behavior of normal and tumor-derived mammary epithelial cells stimulated with WF and stably transduced with miR-223 (Figure 3f). miR-223 overexpression decreased the proliferation of both MCF-10A and BC cells (Figure $4 \mathrm{~b}$ and Supplementary Figure S6A) and also decreased the anchorageindependent growth of BC cells (Figure 4c).

a
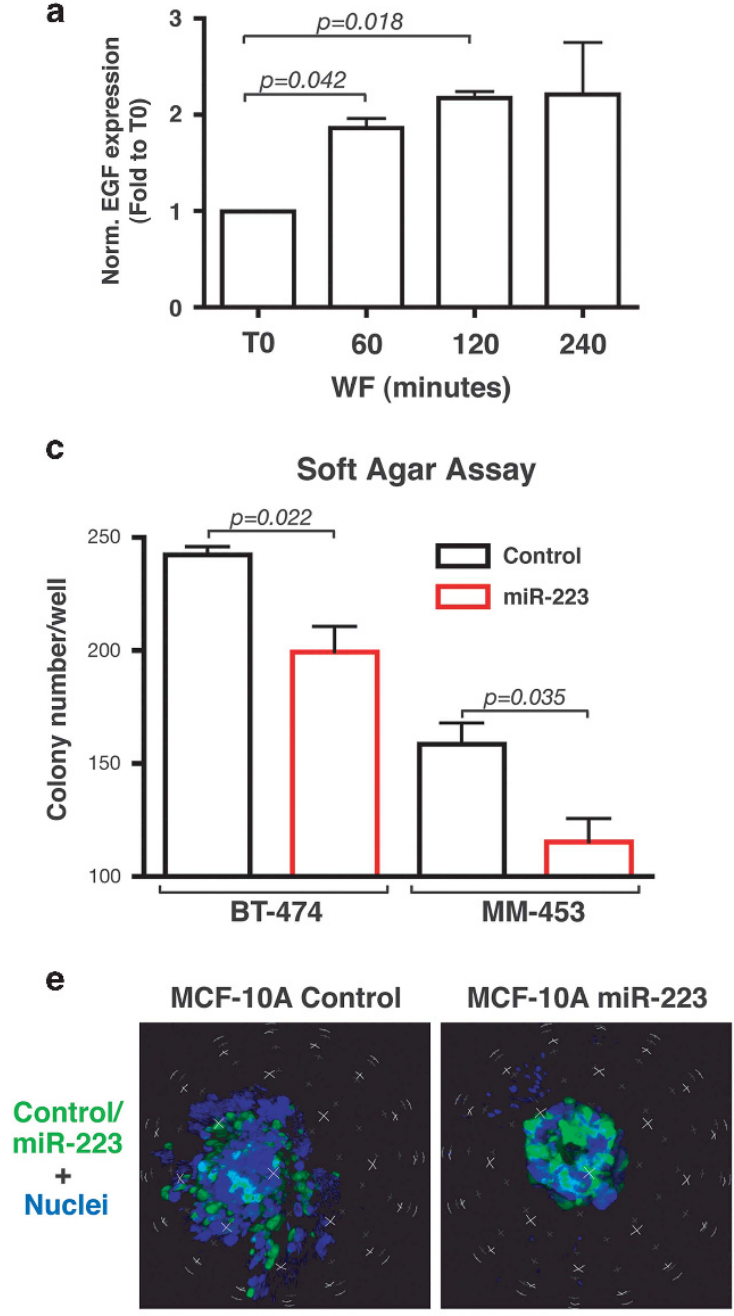

Then, we included cells in three-dimensional (3D) matrices, a condition that more closely resembles the in vivo context. ${ }^{22}$ In all tested cell lines, miR-223 overexpression strongly diminished WFstimulated 3D growth (Figure $4 d$ ). This was particularly evident in MCF-10A cells, which form well-differentiated structures resembling mammary acini (Supplementary Figure S6B). ${ }^{4,23}$ In the presence of WF, MCF-10A displayed a strongly deregulated 3D growth, with a loss of duct-like morphology and acquisition of invasive phenotypes (Figure $4 \mathrm{e}$, left panel). ${ }^{4,24}$ The overexpression of miR-223 was sufficient to rescue their well-ordered duct-like morphology (Figure $4 \mathrm{e}$, right panel). Similar results were obtained using both MCF-7 (estrogen receptor positive (ER+) and progesteron receptor positive $(\mathrm{PgR}+)$, luminal; Figure $4 \mathrm{f}$ and Supplementary Figure S6B) and BT-474 cells (HER2+, luminal; Supplementary Figure S6C), indicating that miR-223 ability to counteract WF-induced cell growth was a general feature observable in different BC subtypes. Thus, expression of miR-223 is able to counteract the pro-tumorigenic stimuli induced by WF.

b

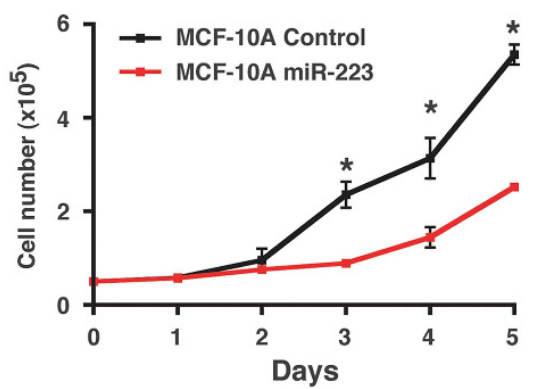

d
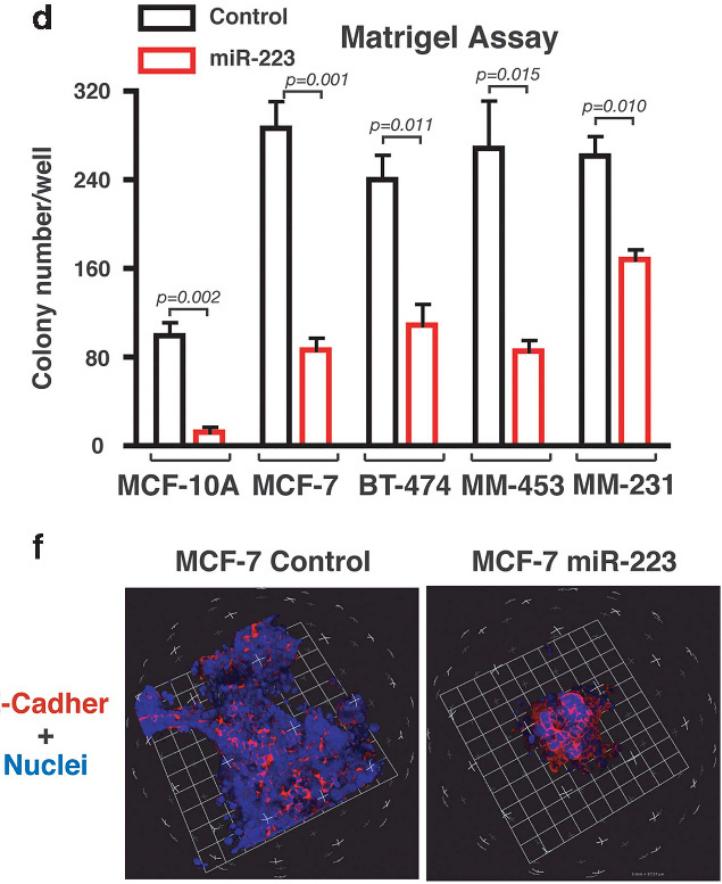

Figure 4. miR-223 controls growth of breast cancer cells, both in two- and three-dimensional (3D) context. (a) qRT-PCR of EGF expression in MDA-MB-231 cells, serum starved and then stimulated with wound fluids for the indicated times. Values were normalized using GAPDH expression and are expressed as fold increase over untreated (T0) cells. (b) Growth curve analysis of MCF-10A cell line stably transduced with control or miR-223 lentiviral vector, plated on day 0 and then counted every day for 5 days, by Trypan Blue exclusion test. (c) Soft agar assay quantification of BT-474 and MM-453 cells transduced with control or miR-223 lentiviral vector in the presence of $5 \%$ WF, allowed to grow for 15 days. (d) Colony number quantification of the indicated cell lines, included in 3D matrigel in presence of 5\% WF and allowed to grow for 15 days. (e, f) Representative 3D reconstruction of confocal images of MCF-10A (e) or MCF-7 cells (f) transduced with control or miR-223 lentiviral vectors, included in 3D matrix as in d. (e) MCF-10A cells were stained with propidium iodide (PI) to visualize the nuclei (blue). EGFP expression (green) was used to identify the control or miR-223 transduced cells. (f) MCF-7 cells were stained for E-Cadherin (red) and nuclei (PI, blue). Data of all graphs represent the mean ( \pm s.d.) of two/three independent experiments performed in duplicates. The Student's $t$-test or Mann-Whitney tests have been used for statistical analysis, as appropriate. ${ }^{*} P \leqslant 0.01$. 
miR-223 controls EGF production and cell proliferation in normal mammary epithelial cells

In contrast with what observed in MCF-10A cells, growth in 3D matrix of human mammary epithelial cells (HMEC) is not dramatically altered by WF. ${ }^{4}$ This observation prompted us to evaluate miR-223 levels in several normal and tumor-derived mammary epithelial cells. HMEC expressed miR-223 at threefold higher level than MCF-10A and MCF-12A, two fibroadenoma- derived cell lines, and up to 20-fold higher than most of other malignant BC cell lines (Supplementary Figure S7A).

To test the effects of miR-223 downmodulation in normal cells, we generated stable anti-miR-223 HMEC cells ${ }^{25}$ (Supplementary Figure S7B). Downmodulation of miR-223 led to increased EGF expression and to a moderate but consistent increase in EGFR-Y1068 phosphorylation (Figures 5a and b). More importantly, anti-miR-223 HMEC displayed increased growth both in two- a

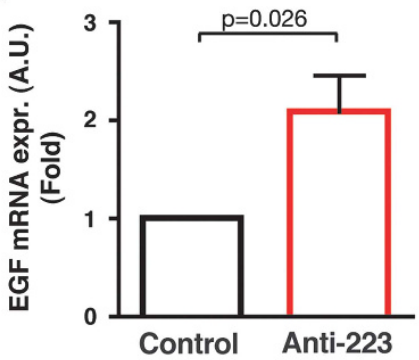

b

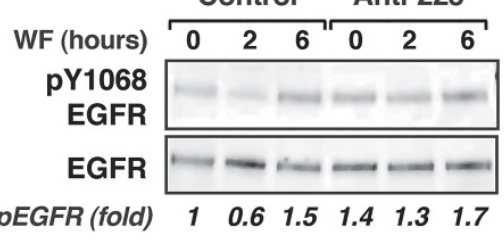

C

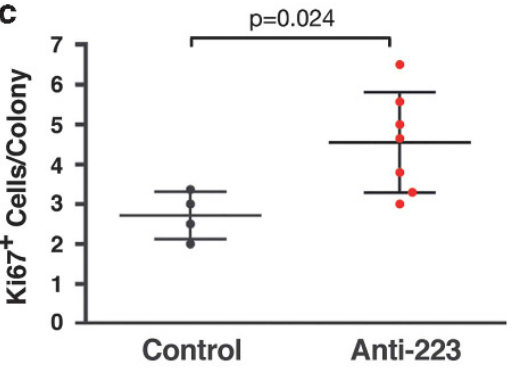

d

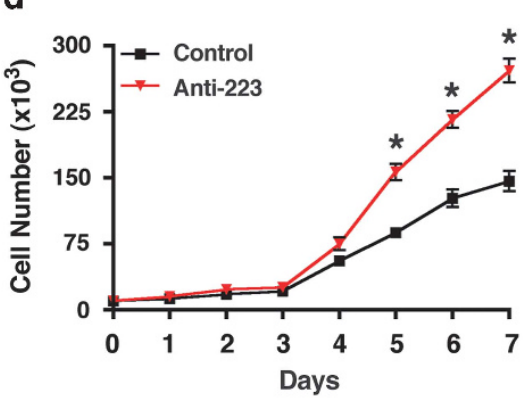

HMEC Control

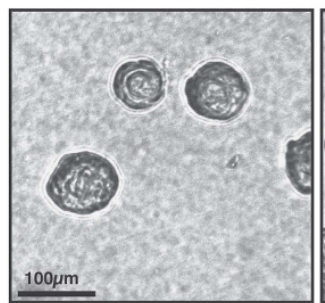

HMEC Anti-223

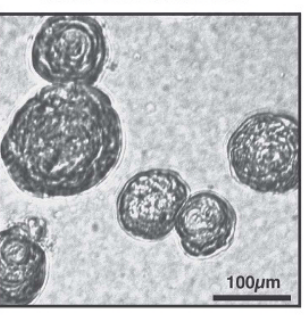

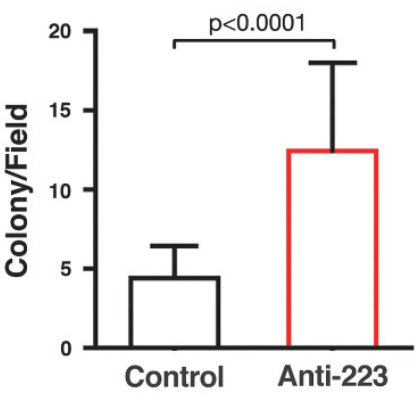

f

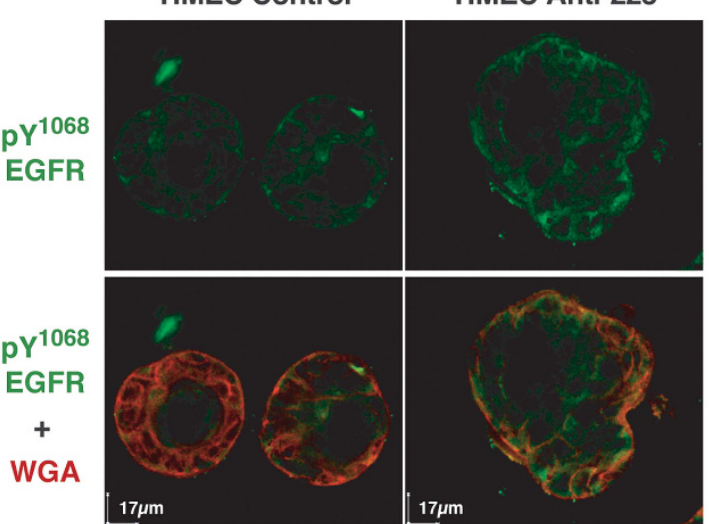

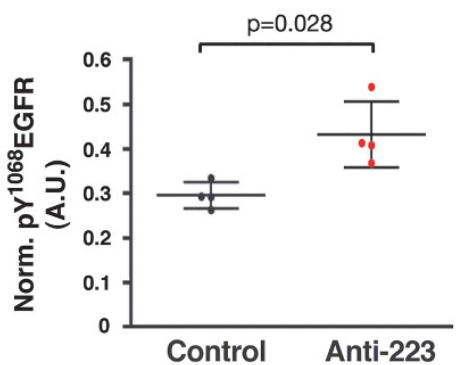

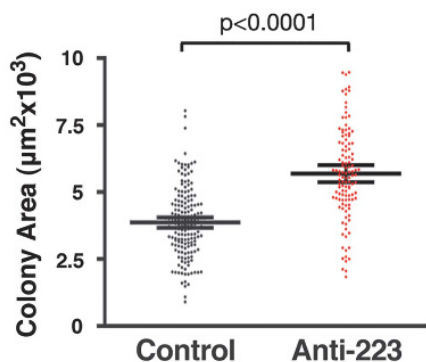

Figure 5. Down modulation of miR-223 expression in HMEC increases EGF signaling and promotes deregulated 3D growth. (a) qRT-PCR of EGF in HMEC control-transfected (black bar) or stably overexpressing anti-miR-223 (red bar). Values were normalized using GAPDH expression and are expressed as fold increase over the expression level of control cells. (b) Western blot analysis of pY1068 EGFR and total EGFR expression in lysates from HMEC control or stably overexpressing anti-miR-223, serum starved and then stimulated with WF for the indicated times. Numbers below represent the quantification of pY1068 EGFR, expressed as fold increase over the control at T0. (c) Graph reports quantification of Ki67-positive cells/colony/field in control and anti-miR-223 transduced HMEC included in 3D matrix and allowed to grow for 7 days. Four and seven fields were scored for control and anti-miR-223 transduced HMEC, respectively. Representative images of 3D confocal reconstruction of HMEC cells are reported in Supplementary Figure 7c. (d) Growth curves of HMEC, control-transfected or stably overexpressing anti-miR-223, plated on day 0 and then counted every day for 7 days by Trypan Blue exclusion test. Data of all graphs represent the mean ( \pm s.d.) of two independent experiments, performed in duplicates. (e) Growth in 3D matrigel of HMEC control-transfected or stably overexpressing anti-miR-223. On the left, representative contrast phase images of the colonies after 7 days of culture are shown. On the right, graph reports the quantification of the colony number in control-transfected and anti-miR-223 overexpressing cells. (f) Immunofluorescence analysis of pY1068 EGFR (green) in HMEC control or stably overexpressing anti-miR-223 as in e. WGA (red) was used to label cellular membrane. Graph in the middle shows the quantification of the pY1068 EGFR fluorescence intensity, normalized for WGA expression and reported as arbitrary units (A.U.). Graph on the right reports the quantification of the colony area in control and anti-miR-223 overexpressing cells. Each dot represents one colony. The Student's $t$-test or Mann-Whitney test have been used for statistical analysis, as appropriate. Asterisks indicate significant differences, ${ }^{*} P \leqslant 0.05 ;{ }^{* *} P \leqslant 0.01 ;{ }^{* * *} P \leqslant 0.001$. 
dimensional and 3D contexts, accompanied by increased Ki67 expression (Figures 5c-e and Supplementary Figures S7C and S7D) and EGFR phosphorylation (Figure 5f). Consistent with previous results, ${ }^{4}$ stimulation with WF did not alter the morphology of the 3D colony in control HMEC cells, but abrogation of miR-223 was sufficient to induce a deregulated 3D growth of the same cells (Figure $5 f$ ).

\section{miR-223 expression controls EGFR activation in BC cells}

The above data suggested that miR-223 overexpression counteracted the wound-induced growth of normal and BC cells, at least in part, by interfering with an autocrine/paracrine stimulatory loop involving the EGF-EGFR signaling axis. To verify this hypothesis, we used different inhibitors of EGFR tyrosine kinase activity. Both Gefitinib and Lapatinib effectively counteracted EGFR phosphorylation following WF stimulation, while Erlotinib was less active (data not shown). Treatment with Gefitinib significantly reduced 3D growth of control cells but failed to elicit significant effects on miR-223 overexpressing cells (Figure 6a), corroborating the notion that miR-223 impacted on 3D growth essentially via the regulation of EGF-EGFR pathway. In this setting, Lapatinib (a dual EGFR and HER2 inhibitor) showed a stronger inhibitory effect than Gefitinib, suggesting that formation of EGFR/HER2 heterodimers could be involved (Figure 6b).

Overall, these data demonstrate that miR-223 impaired WF-induced BC cell growth by inhibiting EGF production and, consequently, EGFR activation. In this context, Lapatinib represented the most effective inhibitor of WF-induced 3D growth of $B C$ cells.

EGFR/HER2 inhibition impairs WF-induced activation of STAT3 and p70S6K signaling pathways

Recently, we demonstrated that robust activation of STAT3 and p70S6K signaling pathways is crucial for BC cells to mediate the pro-survival stimuli provided by the post-surgical wound response and to eventually lead to local recurrence formation. $4,8,24,26$ Interestingly, following WF stimulation, Lapatinib partially blocked STAT3 and p70S6K activation, while having only minor effects on AKT and MAPK signaling, in MDA-MB-231 (Figures $6 c$ and d) and in a larger panel of BC cell lines of different subtypes (Supplementary Figures S8A and B). Lapatinib also induced a remarkable increase in apoptosis, even at suboptimal doses, when miR-223 was overexpressed in BC cells (Figure 6e). Hence, miR-223 overexpression significantly increased sensitivity to Lapatinib and this effect was abrogated by the addition of soluble EGF to the culture medium (Figure 6f). In support of these results, we observed that even under basal culture condition phosphorylation levels of STAT3 and p70S6K pathways inversely correlated with miR-223 expression, although more modestly for STAT3 and more robustly for p70S6K (Supplementary Figure S8C).

Overall, the data collected so far demonstrate that, in the post-surgical setting, EGF is produced as a consequence of the wound response and locally promotes activation of EGFR and downstream pro-survival signaling pathways. The upregulation of miR-223 following RT interferes with this positive loop, eventually leading to decreased $\mathrm{BC}$ cell survival.

\section{$\mathrm{RT}$ interferes with wound-induced BC cell growth}

Taken as a whole, our results suggested that RT could prevent wound-induced BC cell growth not only by killing residual tumor cells but also by dampening EGF availability in the local microenvironment. To prove this hypothesis in vivo, we designed the experiment outlined in Figure 7a. Mice, subjected to breast surgery (wound cohort) or not (control cohort), were irradiated $(5 \mathrm{~Gy})$ or not (NIR), using an image-guided precision irradiator (Figure 7a and Supplementary Figure S9A). Only then, all mice were injected with MDA-MB-231 cells and monitored for tumor growth. The act of surgery per se significantly increased BC cell growth in vivo (Figure $7 \mathrm{~b}$ and Supplementary Figure S9B). Importantly, IR completely abrogated the advantage of woundinduced tumor growth (Figure 7b, compare "Wound 5 Gy" with "Wound NIR", and Supplementary Figure S9B). Conversely, when delivered to non-wounded breast tissue, IR resulted in only minor and transient suppression of cancer cell growth (Figure $7 \mathrm{~b}$ and Supplementary Figure S9B). Altogether, these data demonstrate that the post-surgical IR of mammary glands exerts a specific effect on wounded tissue and on wound-induced cancer cell growth.

To verify if these effects were due, at least in part, to miR-223 expression we used the miR-223 knockout (KO) mouse model, recently characterized by others ${ }^{27}$ (Supplementary Figure S9C). Quantitative reverse transcription-PCR confirmed the expression of miR-223 in the mammary gland of wild-type (WT) and miR-223 heterozygous mice and its absence in miR-223 KO mice (Supplementary Figure S9D). We observed that levels of EGF, measured both in serum $(103 \pm 12 \mathrm{pg} / \mathrm{ml}$ in WT versus $149 \pm 41 \mathrm{pg} / \mathrm{ml}$ in miR-223 KO mice) and in mammary glands (Supplementary Figure S9D, black line), were slightly increased in miR-223 KO mice. The higher levels of EGF observed in miR-223 KO mice resulted in slightly higher phosphorylation of EGFR in mammary glands of miR-223 KO mice than in WT littermates (Supplementary Figure S9E), supporting the possibility that miR-223 regulated the EGF/EGFR pathway also under physiological conditions.

As described above, we delivered IORT in mouse mammary gland before cancer cells implantation (Figure 7c and Supplementary Figure S9F) and then monitored tumor appearance over time. For implantation, we used EO771E2 cells, a very aggressive syngenic cell line from spontaneous BC of C57BL/6 mouse. ${ }^{28}$ Absence of miR-223 resulted in significant anticipation of tumor onset ( $P=0.024$ using a paired $t$-test; Figure $7 \mathrm{~d})$ and increased tumor volume (Figure 7e). Interestingly, the evaluation of tumor burden inhibition due to miR-223 expression indicated that the beneficial effects due to miR-223 induction by IORT decreased with time (Figure 7e, green line). This finding further supports that miR-223 expression, induced in the mammary gland by IORT, exerts its anti-tumor growth effects by regulating the immediate wound response.

Peri-surgical treatment with EGFR inhibitors prevents local recurrence formation

The above data suggested that impairing the activation of EGFR at the 'right' time could prevent local tumor regrowth after breast surgery. We thus tested the in vivo efficacy of Lapatinib administered with a peri-operative treatment schedule, using a mouse model of BC recurrence. ${ }^{8,26}$ MDA-MB-231 cells were injected in mammary fat pad and breast tumor mass allowed to grow up to $\sim 200 \mathrm{~mm}^{3}$. Mice were then randomly divided in four groups, one sham-treated and the others treated with Lapatinib, at 3 different doses, for 3 consecutive days (day - 1, day 0 and day +1 , with respect to surgery). Tumors were surgically removed at day 0 and appearance of local recurrence was monitored over a 7 weeks-long follow-up.

Untreated mice developed local recurrence in 60-70\% of injected sites, in accordance with previous observation. ${ }^{8,26}$ No relevant difference with control mice was detected when the lowest dose of Lapatinib was used (Figure 7f). However, treatment with Lapatinib at doses that efficiently inhibited EGFR activation in vivo resulted in strong reduction of recurrence formation, rendering nearly $90 \%$ of the mice relapse free, when used at $150 \mathrm{mg} / \mathrm{kg}(n=10 ; P=0.005$ in log-rank test; hazards ratio $=9.17$; $95 \%$ confidence interval $1.562-12.83$ ) and $70 \%$ relapse free, when used at $100 \mathrm{mg} / \mathrm{kg} \quad(n=10 ; P=0.04$ in log-rank test; hazards 
ratio $=3 ; 95 \%$ confidence interval 1.043-7.778; Figures $7 f$ and $g$ and Supplementary Figures S9G and $\mathrm{H}$ ).

Overall, these data demonstrated that 3 days of peri-surgical treatment with Lapatinib are sufficient to efficiently suppress BC recurrence.

\section{DISCUSSION}

Here, we have investigated the crosstalk existing between residual BC cells and the post-surgery tumor microenvironment and how RT may interfere with this crosstalk. Our experiments demonstrate that application of RT to the tumor bed, immediately after tumor

b

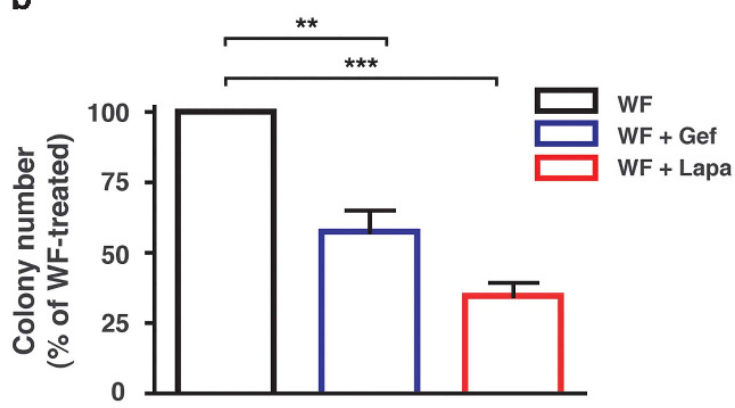

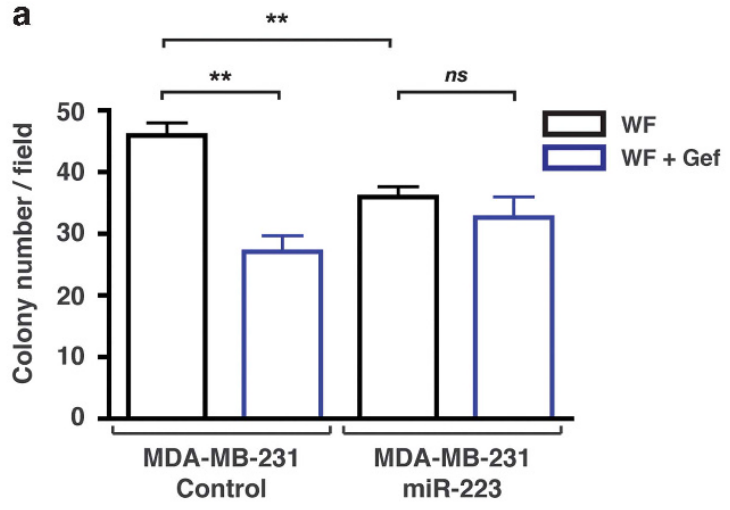

WF +
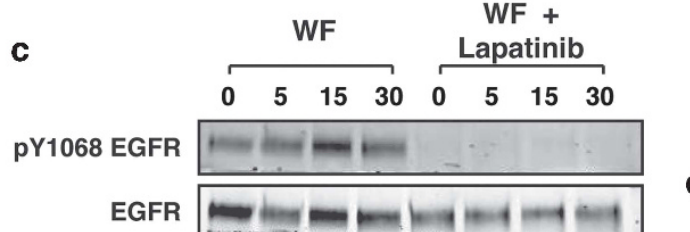

pY705 STAT3

STAT3

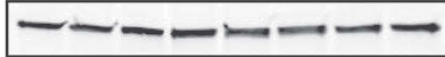

pT389 p70

p70
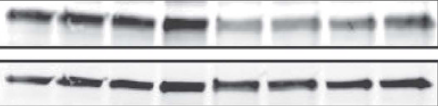

pS235/236 S6

pS240/244 S6

S6
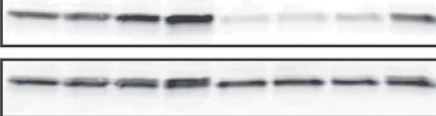

pT202/Y204 ERK1/2

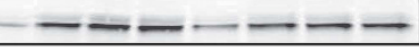

ERK1/2

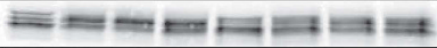

pS473 AKT

AKT

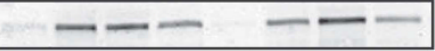

Vinculin

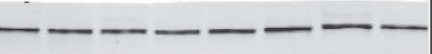

e

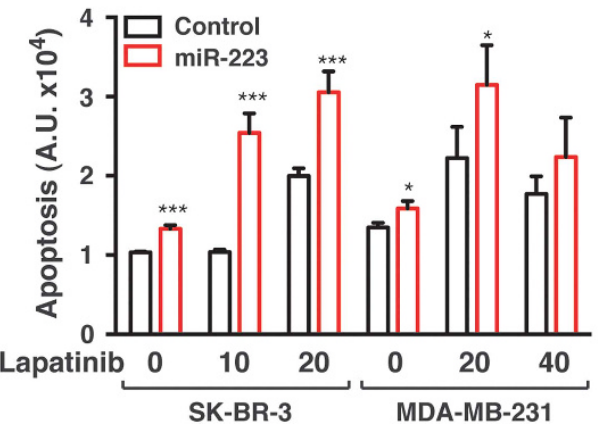

d

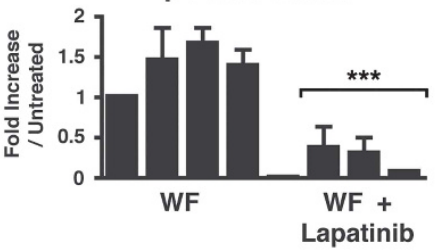

pS240/244 S6
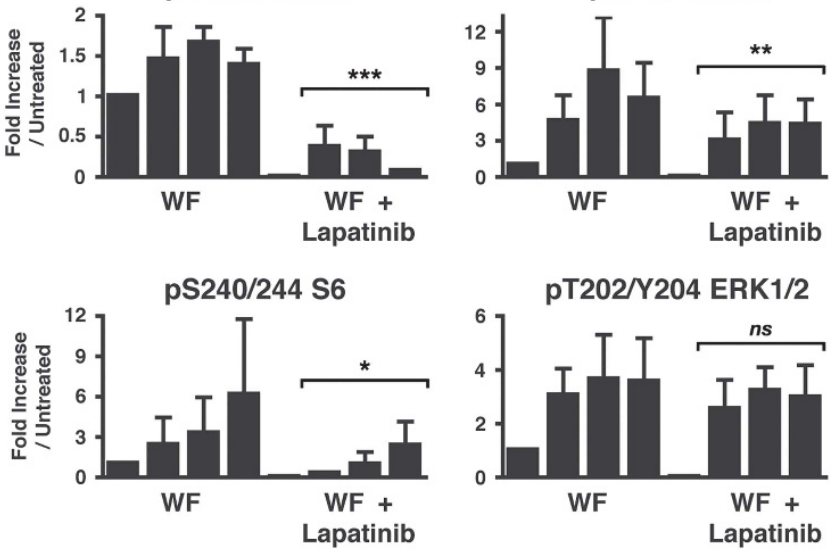

$f$

Cell Line

MDA-MB-231 CTR

MDA-MB-231 miR-223

MDA-MB-231 miR-223 + EGF

SKBR-3 CTR

SKBR-3 miR-223

SKBR-3 miR-223 + EGF

MCF-7 CTR

MCF-7 miR-223

MCF-7 miR-223 + EGF

MDA-MB-453 CTR

MDA-MB-453 miR-223

MDA-MB-453 miR-223 + EGF
IC 50

$46.0 \mu \mathrm{M}$

$9.7 \mu \mathrm{M}$

$46.5 \mu \mathrm{M}$

$17.0 \mu \mathrm{M}$

$0.1 \mu \mathrm{M}$

$21.0 \mu \mathrm{M}$

$24.0 \mu \mathrm{M}$

$8.0 \mu \mathrm{M}$

$14.5 \mu \mathrm{M}$

$33.0 \mu \mathrm{M}$

$1.5 \mu \mathrm{M}$

$24.5 \mu \mathrm{M}$ 
removal, affects the tissue wound-healing response. A large body of literature exists on the possible unwanted and harmful effects of RT. These include onset of cardiac toxicity, appearance of second tumors and, also, the paradoxical stimulation of tumor cell growth, via stimulation of hypoxic conditions, tumor neoangiogenesis and production of pro-metastatic growth factors, such as transforming growth factor- $\beta .^{29-33}$ Whether these harmful effects are related to the dose, the modality and the timing of RT delivery is largely unknown. Previous studies have investigated gene expression changes in $\mathrm{BC}$ at differing time points after $\mathrm{RT},{ }^{34,35}$ but the protocol of IORT has for the first time allowed the investigation of the early effects of RT in vivo, on a wounded peri-tumoral tissue. Exploiting this unique possibility, we specifically chose to focus on miR modifications. This was not only due to the peculiar and pleiotropic nature of $\mathrm{miR}$ and to their high relevance in cancer, but, particularly, for their emerging role as molecules able to act locally and distantly as 'hormones'. ${ }^{14-16}$ We have observed that a group of miR is early modified by RT, and, among them, miR-223 increased following RT with very similar kinetics in humans, mice and cultured mammary epithelial cells, indicating that the observed effect is specific and reproducible. Due to the paucity of material available from patients' 'normal' tissue, we could not unambiguously establish which cellular population (breast epithelial cells, fibroblasts, adipocytes, stromal cells, tumor infiltrating cells...) was the source of RT-induced miR-223 in vivo. However, our experiments highlighted that miR-223 is preferentially expressed in normal mammary gland than in breast tumors or BC cell lines. This finding supports that miR-223 could represent a tumor suppressor, as recently suggested also by others. ${ }^{36}$ Accordingly, our in vitro and in vivo analyses support that, in the context of wound-induced BC growth, expression of miR-223 has the potential to suppress tumor growth, at least in part through the downmodulation of EGF expression and the inhibition of EGF/ EGFR autocrine/paracrine stimulatory loop, initiated by the wound-healing response. Noteworthy, a significant decrease of local EGF expression after IR in patients with head and neck cancer $^{37-39}$ and in mouse bone marrow ${ }^{40}$ has been observed, independently supporting our data.

The relevance of EGF/EGFR pathway in cancer progression is well recognized and extensively investigated. ${ }^{41}$ Local production of EGF following breast surgery is also a well-known molecular event. ${ }^{9}$ The finding that IORT, via immediate miR-223 upregulation, negatively influences this pathway, suggests that designing new therapies to be administered in the peri-operative setting may represent a promising strategy to restrain recurrence in BC patients. Consistently, perioperative administration of Lapatinib was sufficient to efficiently prevent recurrence formation in mice.

In conclusion, our work explores a new signaling axis, taking place in the post-surgery mammary microenviroment that is therapeutically exploitable to restrain recurrence in BC patients. Our findings suggest that proper selection of the 'right' drug for the 'right' patient will not suffice to obtain the most effective treatment for each BC patient. The accurate choice of the most appropriate window of time for drug administration during the course of the disease will also prove crucial for the success of the therapy.

\section{MATERIALS AND METHODS}

Human specimens collection

Two peri-tumoral breast tissue samples were collected from 29 BC patients. For those who underwent only surgery, one specimen was collected immediately after the surgical excision of the tumor and a second one $30 \mathrm{~min}$ after surgery. For those who underwent IORT and surgery, IORT was delivered in the tumor bed for $30 \mathrm{~min}$ after tumor removal. From these patients, one specimen was collected immediately after the surgical excision of the tumor and a second one $30 \mathrm{~min}$ after the end of IORT ( $1 \mathrm{~h}$ from the beginning of IR). Breast tissue specimens were immediately frozen in liquid nitrogen and stored at $-80^{\circ} \mathrm{C}$. Drainage $W F$, harvested from patients for $24 \mathrm{~h}$ after surgery, were collected as previously described. ${ }^{4}$ Unless otherwise specified, pools of WFs ( $>30$ ) from patients who underwent only surgery were used.

\section{Tumor growth in wounded and/or irradiated mice}

Athymic nude mice (Harlan, Foxn $1^{\text {nu }}$, females, 6-8 weeks old) were divided in two cohorts ( $n=10$ for each cohort): one received surgery and one did not. After surgery, the area including the second and third mammary gland was irradiated in half of the mice $(n=5)$ and $3 \mathrm{~h}$ after surgery and/or IR, all mice received an injection of MDA-MB-231 cells in the third mammary gland. Tumor growth was monitored up to 2 weeks. The animals used in this experiments were housed in animal facilities accredited by the Canadian Council on Animal Care and treated in accordance with approved ethical protocols.

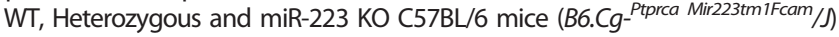
were purchased from The Jackson Laboratory (Sacramento, CA, USA).

\section{Mouse model of IORT}

FVB WT female mice were purchased from Charles River Laboratories (Lecco, Italy). Mice were subjected to surgery followed, or not, by IORT treatment (Carl Zeiss, Oberkochen, Germany), using the spinal applicator. Details can be found in Supplementary Materials and Methods.

\section{Xenograft growth in mouse mammary fat pads and treatment} Athymic nude mice (Harlan, Foxn $1^{\text {nu }}$, females, 6-8 weeks old) were injected with MDA-MB-231 cells. The evaluation of local relapse was performed as previously described. ${ }^{8,26}$ Administration of Lapatinib was performed by oral gavage, following a 3-day 'peri-operative schedule'. Further experimental details are provided in the Supplementary Materials and Methods.

Figure 6. EGFR inhibitors impair wound-induced breast cancer cell growth. (a) Quantification of colony number/field of MDA-MB-231 cells, control-transfected or stably expressing miR-223, included in 3D matrigel in the presence of 5\% WF with (blue bars) or without (black bars) $1 \mu \mathrm{M}$ Gefitinib. Data represent the mean ( \pm s.d.) of two experiments performed in duplicates. (b) MDA-MB-231 cells were included in 3D matrigel in the presence of 5\% WF (black bar) plus $1 \mu \mathrm{m}$ Gefitinib (blue bar), or $2.5 \mu \mathrm{m}$ Lapatinib (red bar), as indicated. Data are expressed as percent of colonies grown in each condition, with respect to the control (only WF) and represent the mean ( \pm s.d.) of two experiments performed in duplicates. (c) Western blot analysis of MDA-MB-231 cells stimulated with WF for the indicated times, with or without Lapatinib, as indicated. The expression of phosphorylated and total forms of EGFR, STAT3, p70S6K, the ribosomal protein S6, ERK and AKT is shown. Vinculin expression was used as loading control. (d) Quantification of pY1068 EGFR, pY705 STAT3, pS240/244 S6 and pT202/Y204 ERK levels in the indicated BC cell lines, stimulated with WF and treated or not treated with Lapatinib. Data represent the mean of three independent experiments and are expressed as fold increase of the indicated phospho-proteins, with respect to the T0 untreated condition. (e) Graph reports apoptotic rate of control (black bars) and miR-223 overexpressing (red bars) SK-BR-3 and MDA-MB-231 cells treated for $24 \mathrm{~h}$ with the indicated doses $(\mu \mathrm{M})$ of Lapatinib, evaluated as activity of caspase 3/7. Data represent the mean of three independent experiments. (f) Evaluation of the IC50 of in the indicated control-transfected or miR-223 overexpressing BC cell lines, in the presence or absence of EGF $(5 \mathrm{ng} / \mathrm{ml})$. IC50 values were calculated by nonlinear regression using the GraphPad Prism software (La Jolla, CA, USA). In all graphs, ${ }^{*} P \leqslant 0.05$; ${ }^{* *} P \leqslant 0.01 ;{ }^{* * *} P \leqslant 0.001 ; \mathrm{ns}$, not significant; indicates a $P \geqslant 0.05$. 
a

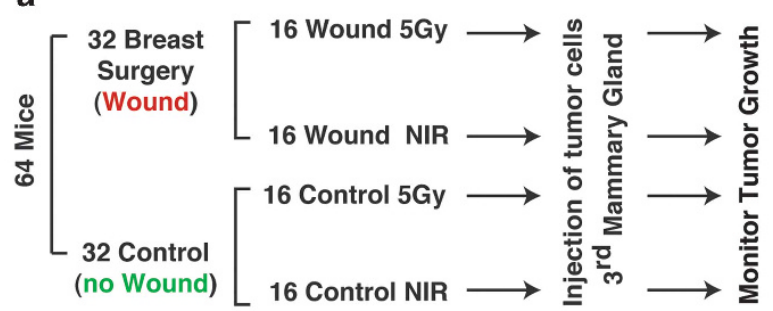

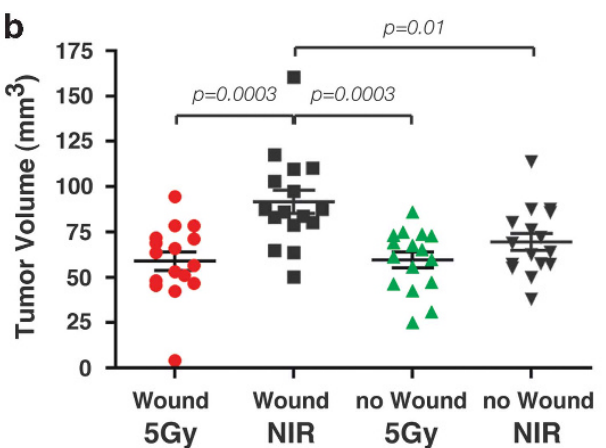

e

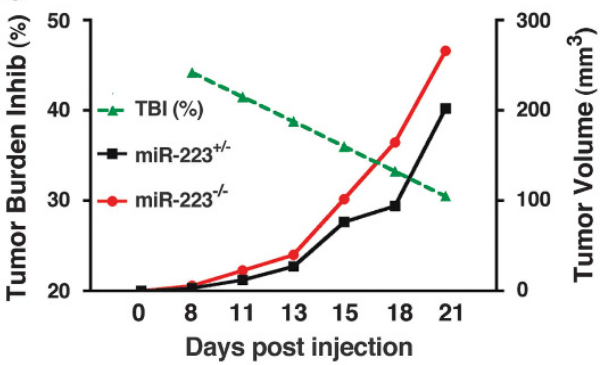

c

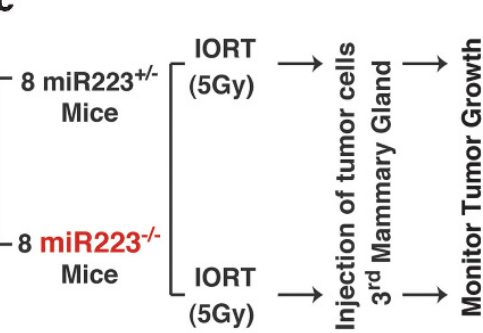

d

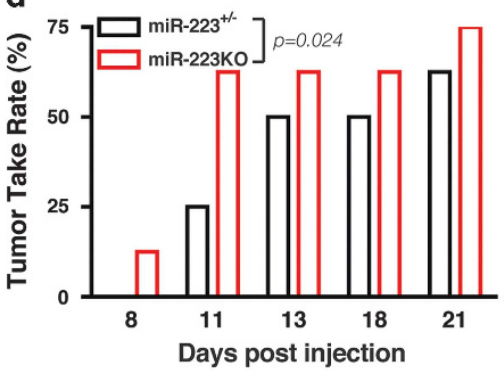

g Vehicle f

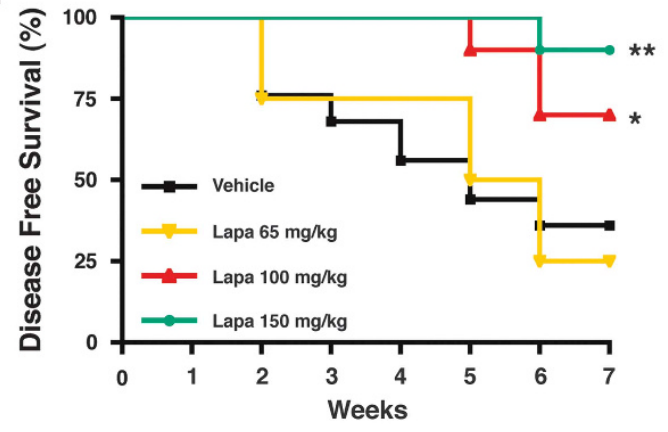

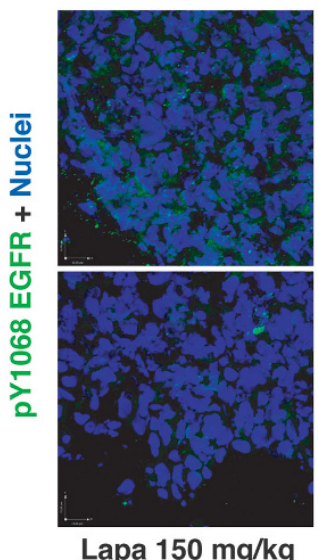

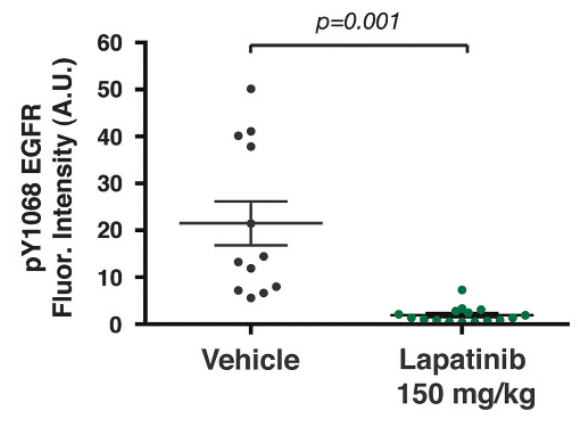

Figure 7. Peri-surgical inhibition of EGFR prevents local recurrence of breast cancer. (a) Schematic representation of the experiment reported in b. Before being all injected with MDA-MB-231 cells, mice were randomized in four cohorts; one subjected to breast surgery and no radiotherapy (wound NIR), one subjected to breast surgery followed by radiotherapy (wound 5 Gy), one subjected only to radiotherapy (control 5 Gy) and one subjected neither to surgery nor to radiotherapy (control NIR). Tumor appearance and growth was then monitored over time. (b) Graph reports the volume of orthotopic xenografts, 13 days post injection of MDA-MB-231 cells, in the different cohorts described in a. Data represent the results from three independent experiments $(n=5+5+6$ in each cohort). The Mann-Whitney test has been used to calculate the significance. (c) Schematic representation of the experiment reported in $\mathbf{d}$ and $\mathbf{e}$. miR- $223^{+/-}$and miR-223 $3^{-1-}$ mice received IORT (5 Gy) with the Intrabeam device. Then all mice were injected with EO771E2 cells. Tumor appearance and growth was then monitored for 3 weeks. (d, e) Tumor take rate (d) and growth (e) in

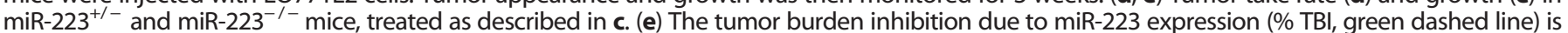
reported. Paired $t$-test has been used to calculate the significance. (f) Disease-free survival analysis of mice subjected to surgery to remove primary tumors (200-300 $\left.\mathrm{mm}^{3}\right)$ and then left untreated $(n=8$, black line) or treated peri-operatively (3 doses) with Lapatinib at $65 \mathrm{mg} / \mathrm{kg}$ ( $n=6$ yellow line) $100 \mathrm{mg} / \mathrm{kg}$ ( $n=10 \mathrm{red}$ line) and $150 \mathrm{mg} / \mathrm{kg}(n=10 \mathrm{green}$ line). Data are reported as percentage of mice that did not developed recurrent disease during 7 weeks of post-surgery follow-up. Kaplan-Meier test has been used to calculate the significance. ${ }^{*} P=0.04 ;{ }^{* *} P=0.005$. (g) Representative confocal images of pY1068 EGFR (green) and nuclei (blue) staining in tumors from mice treated with vehicle or Lapatinib (150 mg/kg) for 3 consecutive days, as evaluated by immunofluorescence analysis. On the right, graph reports the quantification of the mean pY1068 EGFR fluorescence intensity in control $(n=3)$ and Lapatinib-treated $(n=4)$ mice. For each tumor, at least three different fields were analyzed. Mean \pm s.d. and significance are shown.

Cell culture, transduction, soft agar assay and growth in 3D matrices

All cell lines were authenticated by BMR Genomics srl, Padova, Italia, on January 2012, according to Cell ID System (Promega, Madison, WI, USA) protocol and using Genemapper ID Ver 3.2.1 (Applied Biosystems, Foster City, CA, USA), to identify DNA STR profiles. EO771E2 cell line was a kind gift of Dr Qiao Li (University of Michigan Medical Center, Ann Arbor, MI, USA). All other mammary carcinoma cell lines were obtained from ATCC (LGC Standards, Manassas, VA, USA). All in vitro studies were performed in triplicate, unless otherwise specified. A detailed description of all experiments and techniques is reported in the Supplementary Materials and Methods. 
Microarray and molecular biology experiments

Microarray data were submitted using MIAMExpress to the ArrayExpress database (accession number: E-MTAB-1562). A detailed description of experiments, including microarray assay and analysis, protein extraction and western blotting, RNA extraction, quantitative reverse transcription-PCR and miR expression is reported in the Supplementary Materials and Methods.

\section{Statistical analyses}

In each experiment, sample sizes were chosen based on statistical power calculation. The computer software PRISM (version 4, GraphPad, Inc.) was used to make graphs and all statistical analyses. In all experiments, differences were considered significant when $P$-value was $\leqslant 0.05$. Statistical tests are described in detail in the Supplementary Materials and Methods.

\section{Study approval}

WF and peri-tumoral breast tissues were collected at CRO Aviano, Aviano, Italy. Scientific use of biological materials was approved by the Ethic Committee of CRO Aviano. Specific informed consent was obtained from each patient.

All animal experiments were reviewed and approved by the CRO Institutional Animal Care and Use Committee (OPBA) and conducted according to that committee's guidelines.

\section{CONFLICT OF INTEREST}

The authors declare no conflict of interest.

\section{ACKNOWLEDGEMENTS}

This work is dedicated to the memory of $\mathrm{Dr}$ Mauro Gaetano Trovò, who strongly supported this research. We are grateful to the patients who participated to this study. We thank Dr Debora Martinuzzo for her valuable contribution, Sara Benevol for expert technical assistance, Maria Giulia Baldassarre for her useful observations and all members of the SCICC lab for supportive scientific discussions. We thank Dr Qiao Li (University of Michigan Medical Center, USA) for providing EO771E2 cell line and Dr Roberta Maestro (CRO Aviano, Aviano, Italy) for providing HMEC hTERT cells. This work was supported by research funding from Associazione Italiana Ricerca sul Cancro (AIRC) to BB (IG 15902) and to GB (IG 12854); from CRO Intramural Research Grant to GB and SM; from Friuli Exchange Program to SB. SB was a recipient of AIRC-Marie Curie Outgoing International Fellowship. IS was a recipient of AIRC fellowship (ref. 16620).

\section{REFERENCES}

1 Dvorak HF. Tumors: wounds that do not heal. Similarities between tumor stroma generation and wound healing. N Engl J Med 1986; 315: 1650-1659.

2 Arwert EN, Hoste E, Watt FM. Epithelial stem cells, wound healing and cancer. Nat Rev Cancer 2012; 12: 170-180.

3 Baum M, Demicheli R, Hrushesky W, Retsky M. Does surgery unfavourably perturb the 'natural history' of early breast cancer by accelerating the appearance of distant metastases? Eur J Cancer 2005; 41: 508-515.

4 Belletti B, Vaidya JS, D'Andrea S, Entschladen F, Roncadin M, Lovat F et al. Targeted intraoperative radiotherapy impairs the stimulation of breast cancer cell proliferation and invasion caused by surgical wounding. Clin Cancer Res 2008; 14: 1325-1332.

5 Demicheli R, Valagussa P, Bonadonna G. Does surgery modify growth kinetics of breast cancer micrometastases? Br J Cancer 2001; 85: 490-492.

6 Demicheli R, Retsky MW, Hrushesky WJM, Baum M. Tumor dormancy and surgerydriven interruption of dormancy in breast cancer: learning from failures. Nat Clin Pract Oncol 2007; 4: 699-710.

7 Demicheli R, Retsky MW, Hrushesky WJM, Baum M, Gukas ID. The effects of surgery on tumor growth: a century of investigations. Ann Oncol 2008; 19: 1821-1828.

8 Segatto I, Berton S, Sonego M, Massarut S, D'Andrea S, Perin T et al. Inhibition of breast cancer local relapse by targeting p70S6 kinase activity. J Mol Cell Biol 2013; 5: 428-431.

9 Tagliabue E, Agresti R, Carcangiu ML, Ghirelli C, Morelli D, Campiglio M et al. Role of HER2 in wound-induced breast carcinoma proliferation. Lancet 2003; 362: 527-533.
10 Early Breast Cancer Trialists' Collaborative Group (EBCTCG), Darby S, McGale P, Correa C, Taylor C, Arriagada R et al. Effect of radiotherapy after breast-conserving surgery on 10-year recurrence and 15-year breast cancer death: meta-analysis of individual patient data for 10,801 women in 17 randomised trials. Lancet 2011, 378: 1707-1716.

11 Vaidya JS, Joseph DJ, Tobias JS, Bulsara M, Wenz F, Saunders C et al. Targeted intraoperative radiotherapy versus whole breast radiotherapy for breast cancer (TARGIT-A trial): an international, prospective, randomised, non-inferiority phase 3 trial. Lancet 2010; 376: 91-102.

12 Vaidya JS, Wenz F, Bulsara M, Tobias JS, Joseph DJ, Keshtgar M et al. Risk-adapted targeted intraoperative radiotherapy versus whole-breast radiotherapy for breast cancer: 5-year results for local control and overall survival from the TARGIT-A randomised trial. Lancet 2014; 383: 603-613.

13 Liu C-G, Calin GA, Meloon B, Gamliel N, Sevignani C, Ferracin M et al. An oligonucleotide microchip for genome-wide microRNA profiling in human and mouse tissues. Proc Natl Acad Sci USA 2004; 101: 9740-9744.

14 lorio MV, Croce CM. MicroRNA dysregulation in cancer: diagnostics, monitoring and therapeutics. A comprehensive review. EMBO Mol Med 2012; 4: 143-159.

15 Inui M, Montagner M, Piccolo S. miRNAs and morphogen gradients. Curr Opin Cell Biol 2012; 24: 194-201.

16 Redis RS, Calin S, Yang Y, You MJ, Calin GA. Cell-to-cell miRNA transfer: from body homeostasis to therapy. Pharmacol Ther 2012; 136: 169-174.

17 Metheetrairut C, Slack FJ. MicroRNAs in the ionizing radiation response and in radiotherapy. Curr Opin Genet Dev 2013; 23: 12-19.

18 Armenia J, Fabris L, Lovat F, Berton S, Segatto I, D'Andrea S et al. Contact inhibition modulates intracellular levels of miR-223 in a p27kip1-dependent manner. Oncotarget 2014; 5: 1185-1197.

19 Pulikkan JA, Dengler V, Peramangalam PS, Peer Zada AA, Müller-Tidow C, Bohlander SK et al. Cell-cycle regulator E2F1 and microRNA-223 comprise an autoregulatory negative feedback loop in acute myeloid leukemia. Blood 2010; 115: 1768-1778.

20 Hynes NE, Lane HA. ERBB receptors and cancer: the complexity of targeted inhibitors. Nat Rev Cancer 2005; 5: 341-354.

21 Shao H, Cheng HY, Cook RG, Tweardy DJ. Identification and characterization of signal transducer and activator of transcription 3 recruitment sites within the epidermal growth factor receptor. Cancer Res 2003; 63: 3923-3930.

22 Yamada KM, Cukierman E. Modeling tissue morphogenesis and cancer in 3D. Cell 2007; 130: 601-610.

23 Debnath J, Muthuswamy SK, Brugge JS. Morphogenesis and oncogenesis of MCF-10 A mammary epithelial acini grown in three-dimensional basement membrane cultures. Methods 2003; 30: 256-268.

24 Segatto I, Berton S, Sonego M, Massarut S, Fabris L, Armenia J et al. p70S6 kinase mediates breast cancer cell survival in response to surgical wound fluid stimulation. Mol Oncol 2014; 8: 766-780.

25 Haraguchi T, Ozaki Y, Iba H. Vectors expressing efficient RNA decoys achieve the long-term suppression of specific microRNA activity in mammalian cells. Nucleic Acids Res 2009; 37: e43.

26 Segatto I, Berton S, Sonego M, Massarut S, Perin T, Piccoli E et al. Surgery-induced wound response promotes stem-like and tumor-initiating features of breast cancer cells, via STAT3 signaling. Oncotarget 2014; 5: 6267-6279.

27 Johnnidis JB, Harris MH, Wheeler RT, Stehling-Sun S, Lam MH, Kirak O et al. Regulation of progenitor cell proliferation and granulocyte function by microRNA-223. Nature 2008; 451: 1125-1129.

28 Wang SH, Lu L, Fan Y, Wicha MS, Cao Z, Chang AE et al. Characterization of a novel transgenic mouse tumor model for targeting HER2+ cancer stem cells. Int J Biol Sci 2013; 10: 25-32.

29 Schmitz KH, Prosnitz RG, Schwartz AL, Carver JR. Prospective surveillance and management of cardiac toxicity and health in breast cancer survivors. Cancer 2012; 118: 2270-2276.

30 Berrington de Gonzalez A, Gilbert E, Curtis R, Inskip P, Kleinerman R, Morton L et al. Second solid cancers after radiation therapy: a systematic review of the epidemiologic studies of the radiation dose-response relationship. Int J Radiat Oncol Biol Phys 2013; 86: 224-233.

31 Begg AC, Stewart FA, Vens C. Strategies to improve radiotherapy with targeted drugs. Nat Rev Cancer 2011; 11: 239-253.

32 Kuonen F, Secondini C, Rüegg C. Molecular pathways: emerging pathways mediating growth, invasion, and metastasis of tumors progressing in an irradiated microenvironment. Clin Cancer Res 2012; 18: 5196-5202.

33 Barcellos-Hoff MH, Park C, Wright EG. Radiation and the microenvironment tumorigenesis and therapy. Nat Rev Cancer 2005; 5: 867-875.

34 Helland A, Johnsen H, Frøyland C, Landmark HBK, Saetersdal AB, Holmen MM et al. Radiation-induced effects on gene expression: an in vivo study on breast cancer. Radiother Oncol 2006; 80: 230-235. 
35 Halvorsen AR, Helland A, Fleischer T, Haug KM, Grenaker Alnaes GI, Nebdal D et al. Differential DNA methylation analysis of breast cancer reveals the impact of immune signaling in radiation therapy. Int J Cancer 2014; 135: 2085-2095.

36 Pinatel EM, Orso F, Penna E, Cimino D, Elia AR, Circosta P et al. miR-223 is a coordinator of breast cancer progression as revealed by bioinformatics predictions. PLoS One 2014; 9: e84859.

37 Christensen ME, Hansen HS, Poulsen SS, Bretlau P, Nexo E. Immunohistochemical and quantitative changes in salivary EGF, amylase and haptocorrin following radiotherapy for oral cancer. Acta Otolaryngol 1996; 116: 137-143.

38 Dumbrigue HB, Sandow PL, Nguyen KH, Humphreys-Beher MG. Salivary epidermal growth factor levels decrease in patients receiving radiation therapy to the head and neck. Oral Surg Oral Med Oral Pathol Oral Radiol Endod 2000; 89: 710-716.

39 Epstein JB, Gorsky M, Guglietta A, Le N, Sonis ST. The correlation between epidermal growth factor levels in saliva and the severity of oral mucositis during oropharyngeal radiation therapy. Cancer 2000; 89: 2258-2265.
40 Doan PL, Himburg HA, Helms K, Russell JL, Fixsen E, Quarmyne M et al. Epidermal growth factor regulates hematopoietic regeneration after radiation injury. Nat Med 2013; 19: 295-304.

41 Arteaga CL, Engelman JA. ERBB receptors: from oncogene discovery to basic science to mechanism-based cancer therapeutics. Cancer Cell 2014; 25: 282-303

c) (1) This work is licensed under a Creative Commons AttributionBY NA SA Commercial-ShareAlike 4.0 International License. The images or other third party material in this article are included in the article's Creative Commons license, unless indicated otherwise in the credit line; if the material is not included under the Creative Commons license, users will need to obtain permission from the license holder to reproduce the material. To view a copy of this license, visit http:// creativecommons.org/licenses/by-nc-sa/4.0/

Supplementary Information accompanies this paper on the Oncogene website (http://www.nature.com/onc) 